\title{
Verfahren der Texterschließung und Interpretation gezeigt an der Geschichte von Michael Ende Das Haus an der Peripherie
}

\author{
Pavel Váňa
}

This article would like to present a concept for a project-oriented, hands-on learning literature class. The first part examines the general procedures and possibilities related to this approach, whereas the second part shows precise practical methods in reference to Michael Ende's short story Das Haus an der Peripherie.

German literature $-20^{\text {th }}$ century - Michael Ende - project-oriented teaching - literature class

Dieser Artikel will anhand der Geschichte von Michael Ende Das Haus an der Peripherie einige Konzepte zum sowohl rezeptionsorientierten als auch handlungs- und projektorientierten Literaturunterricht veranschaulichen. Im ersten Teil werden dabei allgemeine theoretische Überlegungen zu den einzelnen Verfahren einer Texterschließung und Interpretation dargelegt, während der zweite Teil über konkrete, am Text verankerte Methoden referiert.

Deutsche Literatur - 20. Jahrhundert - Michael Ende - Literaturunterricht Texterschließung - handlungs- und produktionsorientiert

\section{Einleitung}

Das Anliegen dieses Artikels ist es, einige didaktische Herangehensweisen an einen literarischen Text aufzuzeigen, wobei keine Wertung der besprochenen Verfahren angestrebt wird. Die einzelnen Texterschließungs- und Interpretierungsoptionen ${ }^{1}$ werde ich im ersten Teil des Artikels vorstellen. Sie werden exemplarisch in einem weiteren Schritt an Hand der Geschichte Das Haus an der Peripherie von Michael Ende im zweiten Teil des Beitrags veranschaulicht und auf ihre Durchführbarkeit im Rahmen des Literaturunterrichts hin untersucht.

Der o. g. Text zeichnet sich durch eine transparente Symbolik aus, die agierenden Figuren werden neben ihrem unmissverständlich interpretierbaren Handeln durch deutlich erkennbare Merkmale gekennzeichnet, die ihren Charakter zusätzlich illustrieren. Es gibt verhältnismäßig sichtbare Zäsuren im Erzählfluss der

1 Als Einleitung des Kapitels Verfahren der Texterschließung vermerken Ulf Abraham und Matthis Kepser zu den Begriffen Texterschließung und Interpretation, dass es zwischen diesen Begriffen „[...] weder in literaturtheoretischer noch in unterrichtspraktischer Hinsicht eine scharfe Trennlinie gibt. ,Interpretieren', wird es literaturwissenschaftlich gedacht, setzt zwar mehr als ,Erschließen' ein souveränes Subjekt voraus, das [...] die Deutungshoheit über einen Text besitzt, das sich einen Überblick über Inhalt, Struktur und zu lösende Verständnisprobleme verschafft hat [...]. Aber literaturdidaktisch und unterrichtspraktisch ist hier [...] von einem Prozess auszugehen, in dem sich texterschließende und interpretierende Tätigkeiten ergänzen und überlappen “ (Abraham und Kepser 2009: 220). 
Geschichte: M. Ende bedient sich einer klaren Symbolik, die unter Zuhilfenahme von grundlegendem Wissen über die deutsche Geschichte der wilhelminischen Epoche und insbesondere der Zwischenkriegszeit leicht eruierbar ist. So beschaffen, eignet sich der Text dieser Geschichte sehr gut zu einer Analyse und Interpretation in den Literaturseminaren des Hochschulstudiums DaF.

In der folgenden Studie wird ein Unterrichtskonzept für das Literaturseminar im dritten Semester des Bakkalaureatsstudiums an der Pädagogischen Fakultät der Masaryk-Universität Brünn vorgestellt. Im ersten Teil des Beitrags werden einige relevante Modelle des Literaturunterrichts erwähnt und die Struktur eines als Unterrichtsbegleitung und -ergänzung gestalteten E-Learning-Kurses kurz behandelt. Die Arbeitsaktivitäten im Seminar werden zum einen im Präsenzunterricht im Umfang von vier Unterrichtseinheiten realisiert, zum anderen in der Internet-Arbeit in den Modulen und einzelnen Moodle-Themen des zur Ergänzung dieses Seminars erstellten Moodle-Kurses. Als ein Moodle-Thema werden in diesem Artikel die einzelnen Teile, Segmente oder Kapitel eines Moodle-Kurses verstanden, nicht der Begriff Thema in seiner im literaturwissenschaftlichen Bereich üblichen Bedeutung. Die Arbeitsabfolge wird durch die einzelnen Themen eines E-Learning-Kurses (siehe weiter unten) gelenkt.

Der zweite Teil der Studie befasst sich mit den Möglichkeiten einer Umsetzung der im ersten Teil behandelten theoretischen Grundsätze und der praktischen Verwendung des Moodle-Kurses bei der Internet-Arbeit zu Hause.

\section{Modelle des Literaturunterrichts}

Die Lernaktivitäten in einem Literaturseminar erfordern einen intensiven, zeitaufwändigen Arbeitseinsatz sowohl von den Lehrenden als auch von den Lernern. Besonders für die Letzteren ist es nötig, sich durch mehrmaliges Lesen mit der einschlägigen Primärliteratur vertraut zu machen und von den empfohlenen bzw. in eigener Recherche gefundenen Werken der Sekundärliteratur jene auszuwählen, die dazu geeignet sind, die behandelten Teilaspekte des Themas durch relevante zusätzliche Informationen zu erhellen. Die spezifischen Verfahren des Umgangs mit einem literarischen Text bedürfen einer ständigen Vertiefung der Kenntnisse, die sich auf den behandelten literarischen Text beziehen. In der Praxis bedeutet dies, dass man sich ein möglichst komplexes Wissen aus dem Bereich der Bezugswissenschaften zu Eigen machen soll/muss. Es ist sinnvoll, einen Teil der Seminaraktivitäten als Online-Arbeitsmodus im Rahmen eines E-Learning-Kurses zu organisieren. Der Präsenzunterricht kann dadurch wesentlich effektiver gestaltet werden, man kann mehr Freiraum zu Besprechungen der im E-Kurs vorgegebenen Tipps und Anregungen, sowie der von Lernern ausgearbeiteten Materialien schaffen. 


\subsection{Vorentlastung durch einen E-Learning-Kurs}

Der zur Unterrichtsunterstützung erstellte E-Learning-Kurs wird als ein literarischer Online-Kurs im Learning-Management-System Moodle gestaltet und wird den Studierenden bereits vor dem Anfang des Seminars zur Verfügung gestellt. Das folgende Schema zeigt die Struktur der einzelnen Themen des Kurses:

1. Moodle-Thema: Lehrmaterialien

2. Moodle-Thema: Sekundärliteratur (Autor, Werk usw.)

3. Moodle-Thema: Zielsetzungen des Seminars, methodische Schritte (vom Lehrenden ausgearbeitet)

4. Moodle-Thema: Inhaltsangaben und Kommentar des Lehrenden

5. und 6. Moodle-Thema: Ergebnisse des literarischen Gesprächs, Arbeitsthemen der Lerner, die zum großen Teil die Inhalte dieser Themen gestalten

Im ersten Moodle-Thema des Kurses finden die Lerner Lehrmaterialien, die das Seminarziel betreffen - Fachliteraturempfehlungen, Textproben, relevante kurze biographische Angaben und Links zu relevanten themenbezogenen Webseiten.

Das zweite Moodle-Thema, das einige ausschlaggebende Materialien aus dem Bereich der Sekundärliteratur über den betreffenden Autor und sein Werk bereitstellt, dient als Informationspool für die eigenverantwortliche Recherche der Lerner. Es ist empfehlenswert, dem Lerner die konkreten Materialien nicht vorbehaltlos zur Verfügung zu stellen, sondern sein Interesse auf jene Webseiten zu lenken, die er - gemäß seinem Sprachniveau und seinem literarischen Vorwissen - eigenständig verarbeiten kann. Der Unterrichtende muss dabei natürlich sicherstellen, dass die Seiten von entsprechender Qualität sind.

Die Module im dritten Moodle-Thema des E-Kurses sind als Lernplattform konzipiert, in der der Lehrende seine Vorstellungen über die Zielsetzungen des Seminars und die methodischen Schritte entwirft, die im Präsenzunterricht beachtet werden sollen. Es ist ratsam, dass der Lehrende in diesem Abschnitt anhand von kurz gefassten Textseiten konkrete Tipps zur Verfassung der Inhaltsangabe bzw. des 
Précis-Schreibens ${ }^{1}$ den Lernern zur Verfügung stellt, indem er z. B. die Lerner auf die wichtigsten inhaltlichen Stationen der behandelten Geschichte aufmerksam macht und die wesentlichen Punkte markiert, die bei der Inhaltsangabe (bei dem Précis-Schreiben) unter keinen Umständen weggelassen werden dürfen.

Das vierteMoodle-Thema istals Vorstufe der Arbeit in der ersten Unterrichtseinheit anzusehen. Hier werden die von den Studierenden ausgearbeiteten Inhaltsangaben bzw. Précis-Schreiben abgegeben. Um die Lerner zur weiteren Auseinandersetzung mit dem Text zu ermutigen, liest der Lehrer die abgegebenen Arbeiten durch und macht in einem kurzen Kommentar den Lerner auf eventuelle Defizite bei seiner Textwiedergabe aufmerksam. Diese interaktive Offline-Kommunikation zwischen Lerner und Lehrer noch vor Beginn des Präsenzunterrichts ist zwar sehr arbeitsaufwändig, trägt jedoch dazu bei, dass die Lerner motivierter mit dem Text arbeiten und sich später während des literarischen Gesprächs sicherer fühlen.

Die Moodle-Themen fünf und sechs des E-Kurses sind für die weitere Arbeit im Sinne des handlungs- und produktionsorientierten Unterrichts vorgesehen. Die Inhalte dieser Themen werden zum großen Teil von den Ergebnissen des literarischen Gesprächs bestimmt, die während des Präsenzunterrichts in das Moodle-Modul ,Textseiten' im fünften Thema eingetragen werden.

\subsection{Inhaltsangabe}

Bereits bei diesem ersten Schritt der Texterschließung ist es angebracht, vorsichtig vorzugehen und je nach der Zielsetzung für die Unterrichtsstunde oder ihre Ausrichtung die Art und Weise der Inhaltsangabe vorzugeben. Bei manchen literarischen Werken laufen die Lerner schnell Gefahr, die mündlichen oder schriftlichen Formen der Inhaltswiedergabe in unangemessen lange Text- oder Erzählgebilde ausufern zu lassen, wobei die Textstruktur der Textvorlage nicht beachtet und der Inhalt mangelhaft wiedergegeben wird (wichtige Textpassagen werden nicht richtig identifiziert, der Inhalt wird konfus dargelegt, überflüssige Details werden überschätzt und - breit ausgeführt - über die Kernaussagen gestellt, während diese lediglich kurz erwähnt oder ggf. völlig unbeachtet bleiben usw.).

\subsection{Précis-Schreiben}

Einen tieferen Einblick in die Struktur des zu analysierenden Textes können die Lerner beim Verfassen eines Précis-Schreibens gewinnen. Diese Art der Textarbeit

1 Die Verfasser eines Précis-Schreibens sollen „eine Vorlage stiltreu auf exakt 1/3 ihrer Länge [...] verkürzen, unter Beibehaltung der Reihenfolge und wesentlicher Elemente der Handlung [...]. Bisweilen wird in didaktischen Handreichungen auch noch verlangt, dass eine eigene Überschrift gefunden wird und direkte Rede in indirekte oder Redebericht umzuwandeln ist. Solches widerspricht aber [...] der Vorgabe, den Stil des Ausgangstextes nachzuahmen" (Abraham und Kepser 2009: 222). 
richtet sich im Unterschied zu einem inhaltssichernden Verfahren nach strikten Regeln und erfordert daher eine textnahe Lesart. Einige Grundsätze, die bei der Lektüre der zu erschließenden Texte zu wahren sind, gehen von den sechs Thesen von Elisabeth K. Paefgen aus. ${ }^{1}$ Erforderlich ist vor allem ein mehrmaliges Lesen der Geschichte durch den Lerner, der bei seinem Versuch, einen Précis zu schreiben, letztendlich darüber entscheiden muss, welche Teile der Geschichte für ein genügendes Gesamtverständnis relevant sind und welche Textpassagen in eigener Bearbeitung gekürzt oder ggf. ausgeblendet werden können.

So sinnvoll für die Orientierung im Text und dessen Aussage diese Methode ist, kann sie dennoch bei der Arbeit in den Literaturseminaren aus verschiedenen Gründen (wie zum Beispiel eine unzureichende Sprachkompetenz der Lerner) entweder gar nicht oder zunächst nur als Ergebnis einer Gruppenarbeit realisiert werden.

\subsection{Literarisches Gespräch in der Unterrichtspraxis}

Obwohl sich die Studierenden bereits vor dem ersten Präsenztreffen mit dem Thema des literarischen Gesprächs vertraut gemacht haben und idealerweise nicht nur die Quellen der Primärliteratur, sondern auch andere Werke, die im E-Kurs empfohlen werden, studiert oder zumindest kurz gelesen haben, ist es schwierig, sie durch einen wohl durchdachten, anregenden Impuls dazu zu bringen, dass sie gleich bei der ersten Unterrichtseinheit während der Diskussion ungehemmt ihre Äußerungen vortragen, wie es Spinner im vierten Punkt seines Schemas zum literarischen Gespräch vorsieht. ${ }^{2}$ Die Sprachkenntnisse der Seminarteilnehmer zeichnen sich oftmals nicht durch das erforderliche Niveau aus, um von vornherein unbesorgt diskutieren zu können. (In Wirklichkeit ist das für die Seminargruppe offiziell deklarierte B2-Sprachniveau nur bei einigen Studierenden des dritten Semesters erreicht.) Daher sollte die Arbeitsform den realen Bedingungen angepasst werden. Es könnten zum Beispiel drei Studierende aufgefordert werden, in Teamarbeit

1 Gemeint sind die Thesen, die Elisabeth K. Paefgen als kurze didaktische Anleitung zur Realisierung eines sinnvollen textnahen Lesens vorgeschlagen hat: „1. Genaues, langsames, gründliches Lesen/ 2. Textnahes Lesen muss gelehrt und gelernt werden/ 3. Textmenge klein halten/4. Mehrmaliges Lesen des Textes/ 5. Lyrische Texte sind am Geeignetsten/6. Wenig lesen - viel denken“ (Paefgen 1998: 14).

2 Spinner präsentierte ein Modell, in dem ein literarisches Gespräch als ein Verfahren der Interpretation von literarischen Texten in sechs Phasen vorgenommen werden kann: „1. Die leitende Person wählt einen durch Mehrdeutigkeit oder Rätselhaftigkeit auffallenden und daher geeigneten Text aus. 2. Durch einen Sitzkreis schafft man ein klares Setting, gekennzeichnet durch Ruhe, Konzentration und kommunikative Symmetrie. 3. Der Text wird zunächst vorgetragen, dann ausgeteilt und noch einmal still gelesen. 4. Die leitende Person gibt durch einen anregenden Impuls allen die Gelegenheit zu einer ersten Äußerung. 5. Damit ist das Gespräch eröffnet, das auch für Irritation und NichtVerstehen offen ist und ausdrücklich dazu einlädt, eigene Erfahrungen auf den Text zu beziehen. 6. Am Ende werden wichtige Verstehensaspekte und Gesprächserfahrungen zusammengefasst" (Spinner 2006: 15). 
Textstellen zu finden, über die man ein literarisches Gespräch führen kann. ${ }^{1}$ Die drei gewählten (in der Regel sprachlich meist versierten) Lerner könnten in der Stunde des literarischen Gesprächs die Rolle des Moderators übernehmen oder als ein ,Lehrerteam' auftreten. Daher wäre es sinnvoll, wenn sie ihren Entwurf eines Gesprächsplans noch vor der Unterrichtseinheit in einer Konsultations-stunde mit ihrer Lehrkraft eingehend diskutieren.

Im literarischen Gespräch ist es wichtig, dass die Studierenden anhand eigener Erfahrungen über komplizierte, überraschende, unerwartete oder kontroverse Stellen in der Geschichte diskutieren. Dabei sollen jedoch alle Fragen nach dem verborgenen Sinn dieser Stellen zunächst vermieden werden. Dem Fremden, Unverständlichen soll man im literarischen Gespräch ohne jeden Klassifizierungsversuch näher kommen, man mobilisiert in dieser Phase lediglich den Sinn für das Phantastische, Geheimnisvolle, Unheimliche, Unergründbare, man lässt die Atmosphäre, die Existenz der Phänomene aus dem Bereich der perzeptiven Wahrnehmung auf sich wirken. Auf der anderen Seite sollte beim literarischen Gespräch nicht nur der assoziative Gedankenstrom eines jeden Teilnehmers präsentiert werden. Auch der Verteidigung eventuell abweichender Standpunkte sollte ein angemessener Raum vorbehalten werden.

Während die zwei von den drei ,Kollegen' des o. g. ,Lehrerteams' das literarische Gespräch leiten, wird über das literarische Gespräch ein Protokoll geführt, das von einem dritten Lerner, der für diese Stunde die Rolle des Unterrichtenden übernommen hat, in die dazu vorgesehene Textseite des fünften Themas des E-Kurses eingetragen wird. Die Textseite mit dem aktuell entstehenden Protokoll wird jedoch nicht an die Tafel projiziert, damit die Aufmerksamkeit der Lerner nicht vom besprochenen Thema abgelenkt wird und sie ungestört weiter arbeiten können.

\subsubsection{Phase der Verarbeitung}

Nach der ersten Präsenzstunde arbeiten die Lerner zu Hause im Online-Regime - sie besuchen die Seiten des E-Kurses. Im fünften Moodle-Thema des E-Kurses finden sie das Protokoll über das literarische Gespräch und machen sich damit vertraut. Um sicherzustellen, dass sich alle Lerner mit dem Output des literarischen Gesprächs kritisch auseinandergesetzt haben, formuliert der Lehrende Fragen zur Kontrolle, die sich auf das Protokoll beziehen. Überdies versucht er aber auch solche Fragen zu formulieren, die auf das nächste Präsenz-treffen vorbereiten und die Aufmerksamkeit der Lerner auf die wichtigen Stellen der Geschichte lenken. Alle Lerner müssen die Fragen beantworten und sie noch vor der nächsten Präsenzstunde im Modul

1 Diese Textstellen sollten eine Diskussion anregen, im Sinne der fünften Phase eines literarischen Gesprächs nach Spinner, also ein Gespräch, „das auch für Irritation und Nicht-Verstehen offen ist und ausdrücklich dazu einlädt, eigene Erfahrungen auf den Text zu beziehen“ (Spinner 2006: 15). 
,Forum` einreichen. Jeder Lerner wird aufgefordert, mindestens eine Antwort eines Kollegen - im selben Modul ,Forum` - zu kommentieren.

Die auf diese Weise innerhalb von kurzer Zeit entstandene Forum-Diskussion über die Outputs des literarischen Gesprächs nützt der Lehrende als eine Quelle von Meinungen, kritischen Stellungnahmen, Reflexionen und Ansichten. In der ForumDiskussion können die Lerner auf das literarische Gespräch zurückkommen und mögliche weitere Reaktionen auf den entsprechenden Seiten im System Moodle zum Ausdruck bringen, die sie in der Präsenzstunde nicht formuliert haben - sei es, dass sie sich aus Zeitgründen nicht mehr zu Wort melden konnten, oder dass ihnen erst später etwas eingefallen ist, was sie für wichtig halten und noch zusätzlich thematisieren wollen. Der Lehrende überprüft die Antworten und Kommentare. Seine Rückmeldung zu interessanten Äußerungen in der virtuellen Debatte kann für die Lerner sowohl ein erwünschtes Feedback, als auch ein nicht zu unterschätzendes Motivationsmoment darstellen.

\subsection{Unterrichtsgespräch}

Das Unterrichtsgespräch erfolgt während des zweiten Präsenztreffens, idealerweise einer Doppelstunde, die verschiedenartig arrangiert werden kann. Der Lehrende kann die Moderation und Durchführung des Gesprächs selbst übernehmen, die Ergebnisse der Forum-Diskussion präsentieren und zu ausgewählten Reaktionen Stellung nehmen.

In der Folge lenkt der Lehrende die Aufmerksamkeit der Lerner auf die Schlüsselstellen der Geschichte und leitet ein Gespräch über ihre möglichen Aussagen in die Wege. Abschließend können Fragen nach der Funktion der behandelten Textstellen für das gesamte Textgefüge diskutiert werden. Die perzeptive Wahrnehmung der Geschichte (literarisches Gespräch) wird um die Dimension des begrifflich-urteilenden (apperzeptiven) Erfassens der hervorgehobenen Textstellen erweitert.

Es können kurz die wichtigsten sprachlichen und stilistischen Merkmale aufgezeigt werden, an manchen Abschnitten lassen sich die Techniken des textnahen Lesens erproben. Nicht zu unterschätzen sind auch die traditionellen Verfahren des hermeneutischen Konzepts von Sinndeutungen, man sollte aber die hermeneutische Methode eher kurz demonstrieren, um eine, Überdeutung' des Textes und die damit verbundenen endlosen Diskussionen zu vermeiden.

In einem alternativ gestalteten Unterrichtsentwurf für das zweite Treffen beteiligen sich die Lerner in größerem Umfang an den Unterrichtsaktivitäten. Die Unterrichtseinheit wird mit kurzen Auftritten der Lerner eingeleitet, in denen sie anhand der selbst erstellten Schemata die relevanten Merkmale der Text- und Formgestaltung der Geschichte präsentieren, die wesentlichen Stellen der Geschichte argumentativ erfassen und dabei versuchen, die Schlüsselstellen (im Sinne des 
hermeneutischen Ansatzes) zu deuten. Allerdings erfordert dieser Unterrichtsentwurf eine moderierende Beteiligung der Lehrkraft (der Lehrende kann z. B. bei den Deutungsversuchen der Lerner auf die Ambiguität eines jeden literarischen Textes und die damit verbundenen Schwierigkeiten eines hermeneutischen Ansatzes hinweisen). Die einzelnen Hilfestellungen des Lehrenden sollten sich jedoch unter keinen Umständen reglementierend auf das von den einzelnen Lernern durchgeführte Vorgehen auswirken.

\subsection{Handlungs- und produktionsorientierter Unterricht}

In der Abschlussphase des zweiten Treffens werden die Lerner in kleine Gruppen aufgeteilt. Jede Gruppe wird aufgefordert, in der folgenden Doppelstunde von einer der Techniken des handlungs- und produktionsorientierten Unterrichts ${ }^{1}$ bei ihrer kreativen Darstellung einer bedeutenden Textstelle der Geschichte Gebrauch zu machen. Der Lehrende entscheidet in Absprache mit den Lernern darüber, welche Stellen der Geschichte neu bearbeitet werden sollen und auf welche Art diese Neubearbeitung erfolgen soll.

Die kreativen Bearbeitungen der Auszüge aus der Geschichte werden in multimedialer Form beim dritten Präsenztreffen dargeboten (dies kann auf konventionelle Art, etwa durch Power-Point Präsentation oder Handout geschehen, aber auch gegebenenfalls als dramatische Darstellung einer Szene). Die nötigen literaturwissenschaftlichen und fachdidaktischen E-Materialien bezüglich der Techniken des handlungs- und produktionsorientierten Unterrichts finden die Lerner im sechsten Thema des Moodle-Kurses in Form von Web-Links, Textdokumenten (im Modul ,Buch') und in den Videoaufnahmen, die in den vorherigen Jahrgängen als Dokumentation über den handlungs- und produktionsorientierten Unterricht aufgenommen wurden. Das sechste Moodle-Thema ist überdies mit den interaktiven Modulen ,Chat' und ,Forum' ausgestattet, durch deren Verwendung die Kommunikation innerhalb der Arbeitsgruppen effektiv verlaufen kann.

\subsection{Aufsatz}

Im letzten Schritt der Unterrichtsreihe schreiben die Lerner an einer kurzen Abschlussarbeit im Umfang von nicht mehr als zwei Normseiten. Die Arbeit wird nach einheitlichen Vorgaben erstellt und hat den Versuch einer finalen Interpretation der behandelten Geschichte zum Ziel. Die Arbeiten werden vom Lehrenden korrigiert und in der letzten Präsenzstunde besprochen.

1 Der handlungs- und produktionsorientierte Ansatz „zielt auf einen Unterricht, in dem sich die Schülerinnen und Schüler nicht nur lesend und analysierend mit einem Text beschäftigen, sondern der sie in literarischen und anderen ästhetischen Ausdrucksformen tätig werden lässt: Sie schreiben erzählend und lyrisch gestaltend zu Texten, sie interpretieren durch szenische Darstellung, sie malen, erarbeiten Vertonungen u. ä." (Spinner 2006a: 247). 


\section{Wege zur Verwirklichung der behandelten Verfahren}

Im praktischen Teil des Beitrags möchte ich-von den o. a. theoretischen Ausführungen ausgehend - auf einige Tipps, Materialien, Verfahren und Unterrichtsstrategien näher eingehen und konkrete Beispiele anführen, die der Auseinandersetzung mit dem Text der Geschichte von Michael Ende gelten. Dabei wird dieselbe strukturelle Reihenfolge eingehalten, wie im ersten - theoretischen - Teil des Beitrags.

\subsection{Der E-Learning-Kurs Michael Ende, Verfahren einer Interpretation}

Im ersten Thema des Kurses werden relevante kurze Angaben über Endes Leben und sein Werk angelegt (beachtet werden dabei aber lediglich literarische Werke Michael Endes, die nicht in den Bereich der Kinder- und Jugendliteratur einzuordnen sind). Die Lerner finden hier einige Textproben, die sich auf die im Unterricht behandelte Geschichte Das Haus an der Peripherie beziehen. Die Textproben werden von einer Wortschatzliste mit tschechischen Äquivalenten begleitet, die das lexikalische Verstehen schwieriger Textstellen erleichtert.

Die im zweiten Moodle-Thema zur Verfügung gestellten Materialien helfen dem Lerner, sich im Angebot der einschlägigen Titel der Sekundärliteratur zu orientieren. Sowohl bei den Literaturempfehlungen als auch bei den angebotenen Links auf themenbezogene Webseiten muss die Qualität und die Relevanz der empfohlenen Materialien gesichert sein. Als Beispiel können folgende Links angeführt werden, die die wesentlichen Ereignisse aus dem Leben Michael Endes und seines Vaters Edgar Endes dokumentieren:

http://www.dafkurse.de/lernwelt/menschen/ende/ende.htm http://www.thienemann.de/me/galerie.htm http://www.michaelende.de/autor/biographie/edgar-ende-und-seine-kunst http://www.edgarende.de/Home.htm

Neben den Links, die Daten unmittelbar zu M. Ende liefern, werden hier auch Materialien gereicht, die sich auf Nachbarwissenschaften der Literaturwissenschaft beziehen und für die Arbeit an der Geschichte von Nutzen sind. Es handelt sich vor allem um Textdokumente aus den Bereichen der Kunstgeschichte, Soziologie, Religion, Politik usw. Der Interessenschwerpunkt liegt dabei auf der Geschichte der Zwischenkriegszeit bzw. der Nachkriegsentwicklung in Europa. Die Materialien der Sekundärliteratur können u. a. dazu verhelfen, neue Einsichten in die Privatsphäre der Familie Ende zu gewinnen, den Einfluss zu verstehen, den die künstlerische Tätigkeit seines Vaters auf Michael Ende ausgeübt hatte, oder sich in die Schwierigkeiten und Ängste hineinzuversetzen, mit denen das Leben der Familie zur Zeit des Nationalsozialismus verbunden war. 
Das dritte Thema des E-Kurses ist dem Lehrenden vorbehalten. Er kreiert die Inhalte des Moodle-Themas teils schon vor dem Beginn des Unterrichts und formuliert sowohl Organisatorisches, (Beschreibung der zeitlichen Abfolge der Seminarreihe, weitere Informationen bezüglich des Unterrichts usw.), als auch konkrete Anleitungen/ Arbeits-aufträge. Bevor die Lerner an der Inhaltsangabe (bzw. am Précis) schreiben, stellt er ihnen eine Textseite zur Verfügung, auf der er die ,Textstationen' der Geschichte zeigt, die in der Inhaltsangabe nicht fehlen dürfen. Für die hier analysierte Geschichte könnten die Textstationen folgende Gestalt annehmen:

- Rahmen der Geschichte (der Untertitel Ein Leserbrief ist dabei zu erwähnen, der Text ist bei weiterer Arbeit nach den textspezifischen Mustern eines Leserbriefs zu prüfen),

- Einstieg in die Geschichte, zeitliche Einordnung, Familie des Erzählers,

- die Figur der Schoaßwalli,

- das Haus - Beschreibung, das Innere des Hauses,

- der Auftrag, die Brüder besuchen das Haus,

- das Erlebnis der Leere,

- Gespräch mit der Schoaßwalli,

- ein gefährliches Wissen, der Traum,

- SS-Leute im Haus,

- Mann in mit Hut und Mantel,

- das Erforschen des Hauses,

- Kriegsende, Vernichtung des Hauses.

Einen Teil der im dritten Thema des E-Kurses gezeigten unterstützenden Unterrichtsmaterialien erarbeitet der Lehrende als Hinweise, Reflexionen, Korrekturen und Ratschläge, die auf die aktuellen Outputs der Präsenzstunden Bezug nehmen. In diesen Materialienbereich gehören u. a. auch die Fragen, die der Lehrende nach dem ersten Präsenzunterricht an die Lerner richtet. Einige davon kann er schon vorformuliert haben, andere ergeben sich jedoch erst aus dem literarischen Gespräch.

Fragen, die das literarische Gespräch reflektieren, könnten lauten:

Wodurch entsteht, das Unheimliche' in der Geschichte?

Wie begegnen die Charaktere der Geschichte dem Unbekannten/Bedroblichen? Gelingt es ihnen, ihre Angst zu überwinden?

Fragen, die schon als Brücke zur zweiten Präsenzstunde verstanden werden könnten: 


\section{Was symbolisiert das Haus?}

Wie reagieren die Kinder und wie die Erwachsenen auf die seltsamen Vorfälle?

Wer war der Mann in Hut und Mantel?

Wie verstehen Sie den Traum von Emil und Joseph?

\subsection{Inhaltsangabe}

Bei dem hier besprochenen Werk von M. Ende ist die Inhaltsangabe als unentbehrlicher Ausgangspunkt für das Verstehen der Geschichte anzusehen. Für das schriftliche oder mündliche Nacherzählen des Textes sind den Lernern möglichst kurze Formulierungen abzuverlangen, um den im Unterkapitel 1.2 beschriebenen Fehlern bei der Inhaltswiedergabe möglichst aus dem Weg zu gehen. Der Auftrag, eine kurze Textzusammenfassung zu erstellen, erfordert von den Lernern eine wiederholte Lektüre des Textes, die Bemühung um ein fehlerfreies Textverstehen ${ }^{1}$ (Erhöhung der Kompetenzen beim Leseverstehen und Sprechen) und letztendlich die Entschlüsselung (also Wahrnehmung) der zugrundeliegenden Textstruktur. Es ist ratsam, dass der Lehrende auf die einzelnen Inhaltsangaben in der ersten Präsenzstunde eingeht und sie dann mit den Lernern kritisch bespricht.

\subsection{Literarisches Gespräch}

In der Geschichte Das Haus an der Peripherie sind folgende Stellen als ein geeigneter Einstieg in die Debatte zu betrachten:

a) Die Leere des Hauses

b) Menschen, bei denen „etwas“ nicht stimmt

c) Der Traum als Reaktion auf ein reales Erlebnis

d) Handeln wider die Angst

ad a): Beim ersten Punkt lautet die Frage: Wie würdest du reagieren, was würdest du füblen, wenn es dir passieren würde, dass du durch ein Haus gehst, das von außen wie ein normales Haus aussieht, das aber keine Innenräume hat? Wenn die Gesprächsteilnehmer nach diesem Punkt befragt werden, ist eine häufige Reaktion: Das kann ich mir nicht vorstellen. Das ,Lehrerteam' muss dann unbedingt in der Lage sein, die Lerner dazu zu bringen, sich in die Rolle der handelnden Figuren zu versetzen und den Versuch zu wagen, ihre Gefühle zu formulieren. Gelingt es den Lehrenden, den Lernern den surrealen Charakter der Situation nahe zu bringen, werden diese dadurch ein wichtiges Grundelement dieser Geschichte verstehen lernen.

1 Mit „Textverstehen“ ist nicht primär an den Sinn der Geschichte gedacht, sondern vor allem an die verschiedenen Deutungsebenen und ihre Bedeutung für die ,Aussage‘ der Geschichte. 
ad b): Analog zum Punkt a) wird auch hier über die eigene Erfahrung mit Menschen, bei denen ,etwas nicht stimmt“ gesprochen. Früher oder später wird man schließlich auf die Schoaßwalli zu sprechen kommen. Konsequenterweise müssten aber die Moderatoren dies verhindern, denn bei dem literarischen Gespräch sollen Gefühle und Wahrnehmungen mobilisiert und formuliert werden, die sich nicht auf eine konkrete Situation/Figur der Geschichte, sondern auf eine dem Typus nach entsprechende allgemeine Situation/Figur beziehen. Man sollte daher möglicherweise nicht von der Schoaßwalli, sondern von einem beliebigen Menschen aus der eigenen Erfahrungswelt erzählen, bei dem ,etwas' nicht in Ordnung zu sein scheint.

ad c): Der Traum bildet einen der zentralen erzählerischen Momente der Geschichte. Durch eine Debatte über den Traum als Reaktion auf ein reales Erlebnis kann man auf die Mechanismen der Wechselwirkung von Traum und Realität aufmerksam werden. Die größte Gefahr der Debatte zu diesem Punkt besteht dabei in einer allzu wissenschaftlichen Zugangsweise zum Thema ,Traum'. Es muss immer wieder der Grundsatz geltend gemacht werden, dass man entweder über die eigenen Erfahrungen spricht oder darüber, was einem in Bezug auf das Thema unverständlich vorkommt bzw. was man für strittig hält.

ad d): Bei diesem Punkt handelt es sich um die Evokation dessen, was man verspürt, wenn man eine Handlung realisiert, deren Durchführung von Angstgefühlen begleitet wird.

\subsection{Unterrichtsgespräch}

Als Einstieg in das Unterrichtsgespräch können die Reaktionen der Lerner auf die Fragen des Lehrenden im Moodle-Forum dienen und ihre Kommentare zu den Antworten der Mitstudenten.

Im zweiten Teil des Unterrichtsgesprächs kann man sich auf die stilistischen und sprachlichen Elemente der Geschichte konzentrieren und z. B. auf die in den Dialogen der Protagonisten auftretenden Abweichungen von der Sprachnorm hinweisen, die Gestaltung der Figurenkonstellation untersuchen, über die mögliche Symbolik der Zeitspanne der erzählten Zeit diskutieren oder die Lerner auf die erzähltechnischen Mittel der als ein Leserbrief getarnten Geschichte aufmerksam machen.

Eine andere Vorgehensweise sieht vor, dass der Lehrende die Schlüsselstellen der Geschichte mit den Lernern bespricht und sie auffordert, diese Stellen zu deuten. Er moderiert ihre Deutungsversuche und insistiert darauf, die geäußerten Vermutungen anhand von zitierten Textstellen zu belegen.

Als Beispiel kann die Textstelle genannt werden, in der ein Mann in Begleitung von zwei SS-Männern in das Haus geführt wird (Ende 2002: 109). Hier kann die Besprechung folgende Punkte berühren: Warum wird der Mann in das Haus geführt, wer ist er, welche Rolle spielt die Schoaßwalli dabei, wie urteilen die Protagonisten 
über diese Szene? Ein anderes Beispiel könnte die bereits im literarischen Gespräch behandelte Textstelle darstellen, in der der Traum der Brüder Emil und Joseph beschrieben wird (Ende 2002: 108-109).

\subsection{Handlungs- und produktionsorientierter Unterricht}

Diese Unterrichtseinheit stellt den Abschluss des Unterrichtszyklus dar. Die Lerner präsentieren ihre kurzen szenischen Darstellungen der wichtigsten Stellen der Geschichte. Je nach Stelle können sie ihre Bearbeitung als Weiterführen von Dialogen gestalten. Die dazu geeigneten Dialoge führen Emil und die Schoaßwalli:

„Da“, sagte sie, „das ist dann für dich.“ [...] Die Schoaßwalli schwang sich aufs Rad, und schon im Wegfahren rief sie zurück: „Besuch mich doch mal, Bub. Du weißt ja, wie man reinkommt.“ (Ende 2002: 107)

Nach diesen Worten zeigt sich Emil sehr besorgt:

„Nicht einmal mir hatte Emil verraten, dass er für sich einen Reserveschlüssel gefeilt hatte in der Absicht, in Abwesenheit der Schoaßwalli das Haus noch einmal zu untersuchen. Nun beunruhigten ihn ihre Abschiedsworte sehr [...].“ (Ende 2002: 107)

Ein von den Lernern erfundenes weitergeführtes Gespräch könnte der Frage gelten, wie die Schoaßwalli wissen konnte, dass Emil heimlich einen Schlüssel angefertigt hat. Auch das Gespräch zwischen den Brüdern Emil und Joseph könnte nach Emils Frage fortgeführt werden:

„Nur eins möcht ich wissen. Warum muss es dann abgesperrt werden, wenn doch sowieso keiner hineinkann? Da ist doch überhaupt nichts." (Ende 2002: 106)

Das beschriebene Konzept lässt sich beliebig erweitern, abhängig von Kreativität, Einsatzfreude und Motivation sowohl der Lerner als auch der Lehrenden. Mögliche Beispiele wären:

- akustische Darstellung (eine Lernergruppe findet oder kreiert eine Hintergrundmusik, passend zum dramatischen Vortrag der diskutierten Textstelle),

- filmisches Äquivalent (die Lernergruppe sucht einen Filmausschnitt, der die Grundstimmung der diskutierten Textstelle widerspiegelt), 
- graphische Darstellung (ausgewählte Situationen werden graphisch in Form einer Collage oder eines Comics umgesetzt),

- Hörspiel (zur Realisierung dieser Art Bearbeitung werden reduzierte Textfassungen herangezogen, die Durchführbarkeit des Vorhabens hängt jedoch von den zeitlichen und technischen Möglichkeiten der Beteiligten $\mathrm{ab})$,

- Textsortenumwandlung (die Geschichte kann als eine andere Textsorte geschrieben werden, z. B. als Interview, Bericht, Monolog u. ä.).

Die o. g. Darstellungsmöglichkeiten sollen dazu dienen, dass die Lerner für die Grundstimmung der Geschichte sensibilisiert werden und dass sie gemäß den Richtlinien des handlungs- und produktionsorientierten Unterrichts Bezugspunkte außerhalb der Textrealität herstellen können.

\section{Schlussbetrachtung}

Die hier skizzierten Mittel, Wege und theoretischen Konzepte einer Arbeit mit/ an einem literarischen Text im Literaturunterricht erheben keinerlei Anspruch, eine vollständige Übersicht von realisierbaren Arbeitstechniken in Bezug auf die Erschließung/Interpretation eines literarischen Textes zu sein. Es geht vielmehr darum, am Beispiel eines kurzen Textes von Michael Ende auf die vielfältigen Möglichkeiten der Gestaltung des Literaturunterrichts hinzuweisen. Die Attraktivität des Unterrichts kann durch die Verbindung von Lernaktivitäten des Präsenzunterrichts mit der Arbeit der Lehrer und Lerner im Online-Modus, u. a. unter Verwendung der oben skizzierten Inhalte in hohem Maße gesteigert werden.

\section{Literaturverzeichnis}

Ende, Michael (2002): Das Gefängnis der Freiheit. München, Goldmann.

Abraham, Ulf / Kepser, Matthis (2009): Literaturdidaktik Deutsch. Eine Einfübrung. Berlin, Erich Schmidt-Verlag.

Spinner, Kaspar H. (2006): Literarisches Lernen. In: Praxis Deutsch. 33/ 200/ 2006. S. 6-16.

Spinner, Kaspar H. (2006a): Handlungs- und produktionsorientierter Literaturunterricht. In: Bogdal, Klaus-Michael / Korte, Hermann (Hg.): Grundzüge der Literaturdidaktik. München. Deutscher Taschenbuch Verlag. S. 247-257.

Paefgen, Elisabeth (1998): Textnahes Lesen. Sechs Thesen aus didaktischer Perspektive. In: Belgrad, Jürgen / Fingerhut, Karlheinz (Hg.): Textnahes Lesen. Annäherungen an Literatur im Unterricht. Baltmannsweiler. Schneider-Verlag Hohengehren. S. 14-23. 